\title{
Universality of the Wigner time delay distribution for one-dimensional random potentials
}

\author{
Christophe Texier ${ }^{\dagger}$ and Alain Comtet ${ }^{\ddagger}$ \\ Laboratoire de Physique Théorique et Modèles Statistiques. \\ Université Paris-Sud. Bât. 100. 91405 Orsay cédex. France.
}

(October 3, 2018)

\begin{abstract}
We show that the distribution of the time delay for one-dimensional random potentials is universal in the high energy or weak disorder limit. Our analytical results are in excellent agreement with extensive numerical simulations carried out on samples whose sizes are large compared to the localisation length (localised regime). The case of small samples is also discussed (ballistic regime). We provide a physical argument which explains in a quantitative way the origin of the exponential divergence of the moments. The occurence of a log-normal tail for finite size systems is analysed. Finally, we present exact results in the low energy limit which clearly show a departure from the universal behaviour.
\end{abstract}

The problem of quantum scattering by chaotic or disordered systems is encountered in many fields ranging from atomic or molecular physics as well as in the scattering of electromagnetic microwaves. Some properties of the scattering process are well captured through the concept of time delay. This quantity, which goes back to Eisenbud and Wigner [7], is related to the time spent in the interaction region by a wave packet of energy peaked at $E$. It can be expressed in terms of the derivative of the $S$ matrix with respect to the energy. In the context of chaotic scattering the approach based on random matrix theory (RMT) provides a statistical description of the time delays. This problem was first studied by a supersymmetric approach 11] and in [13 by using a statistical analysis. This latter work provides a derivation for the one channel case for the different universality classes. Recently it served as a starting point for [3] where the $N$ channel distribution is shown to be given by the Laguerre ensemble of RMT. In spite of its success, such a description by RMT is not entirely satisfactory, in particular it does not apply to strictly one-dimensional systems 10 for which strong localisation effects occur. Furthermore it does not shed much light on the physical mechanisms which are responsible for the universal distribution. In this work we explore another approach by considering the scattering by a one-dimensional random potential. In this case the existence of universal distributions was first conjectured in [6] on the basis of a comparative study of two different models. This was further supported by [16] where the random potential is still of a different kind.

The purpose of this letter is to present a new derivation that accounts for the universality and also to provide a physical picture that explains the origin of the algebraic tail of the distribution in terms of resonances. Further details will be given elsewhere [22]. To begin with, let us briefly recall the model. We consider the Schrödinger equation on the half line $x \geqslant 0$ :

$$
-\frac{\mathrm{d}^{2}}{\mathrm{~d} x^{2}} \psi_{k}(x)+V(x) \psi_{k}(x)=k^{2} \psi_{k}(x)
$$

We assume that $V(x)$ has its support on the interval $[0, L]$ and impose the Dirichlet boundary condition $\psi_{k}(0)=0$. Therefore, for $x \geqslant L$ stationary scattering states of the form

$$
\psi_{k}(x)=\frac{1}{2}\left(\mathrm{e}^{-\mathrm{i} k(x-L)}+\mathrm{e}^{\mathrm{i} k(x-L)+\mathrm{i} \delta(k)}\right)
$$

represent the superposition of an incoming and a reflected plane wave. Since there is only backward scattering, the reflection coefficient $\mathrm{e}^{\mathrm{i} \delta(k)}$ is of unit modulus and the Wigner time delay takes the form $\tau(k) \stackrel{\text { def }}{=} \frac{1}{2 k} \frac{\mathrm{d} \delta(k)}{\mathrm{d} k}$. Such a model with a random potential can be viewed as a model of a disordered sample connected to an infinite lead. Instead of using the invariant embedding method as in [15,14] or stochastic differential equations [8], our starting point is to relate the time delay to the wave function inside the sample. This may be achieved by using the identity

$$
\frac{\mathrm{d}}{\mathrm{d} x}\left(\frac{\mathrm{d} \psi^{*}}{\mathrm{~d} x} \frac{\mathrm{d} \psi}{\mathrm{d} E}-\psi^{*} \frac{\mathrm{d}^{2} \psi}{\mathrm{d} x \mathrm{~d} E}\right)=|\psi|^{2} .
$$

By integration over $[0, L]$ one gets the so-called Smith formula 20]

$$
\tau(k)=\frac{2}{k} \int_{0}^{L} \mathrm{~d} x\left|\psi_{k}(x)\right|^{2}-\frac{1}{2 k^{2}} \sin \delta(k) .
$$

It expresses the time delay as the sum of a dwell time [1] and a term that can be neglected in the high energy limit. Inside the sample, the wave function and its derivative may be written in the form $\psi_{k}(x)=\mathcal{N} \sin \theta(x) \mathrm{e}^{\xi(x)}$ and $\psi_{k}^{\prime}(x)=k \mathcal{N} \cos \theta(x) \mathrm{e}^{\xi(x)}$. The normalisation factor $|\mathcal{N}|=\mathrm{e}^{-\xi(L)}$ is fixed by matching the wave function at $x=L$ with the scattering states (2). We now consider the case where $V(x)$ is a random potential. In this case the growth or decay of the envelope $e^{\xi(x)}$ of the wave function is measured by the Lyapunov exponent $\gamma$ (inverse localisation length $\lambda=\gamma^{-1}$ ). In the high energy limit, the envelope is a slow variable, while the phase 
$\theta(x)$ presents rapid oscillations on a scale $k^{-1}$. Therefore, in the high energy limit one can integrate over the fast variable in (雨) and get:

$$
\tau(k)=\frac{1}{k} \int_{0}^{L} \mathrm{~d} x \mathrm{e}^{2(\xi(x)-\xi(L))} .
$$

This representation of the time delay holds for any realization of the disordered potential. It therefore captures all the statistical properties of $\tau(k)$ once the distribution of $\xi(x)$ is known. Denoting by $x_{c}$ the correlation length of $V(x)$ and assuming that $x_{c}$ and $k^{-1}$ are the smallest length scales of the system, then it was proven in [1] that the variable $\xi(x)$ is a Brownian motion of the form: $\xi(x)=\gamma x+\sqrt{\gamma} W(x)$ where $W(x)$ is a normalised Wiener process $\left(\langle W(x)\rangle=0,\left\langle W(x) W\left(x^{\prime}\right)\right\rangle=\min \left(x, x^{\prime}\right)\right)$. Thus the Lyapunov exponent $\gamma$ controls both the drift and the fluctuations. Using the scaling properties of the Brownian motion then gives the following identity in law:

$$
\tau(k) \stackrel{(\text { law })}{=} \frac{1}{k \gamma} \int_{0}^{\gamma L} \mathrm{~d} u \mathrm{e}^{-2 u+2 W(u)} .
$$

This representation of the time delay as an exponential functional of the Brownian motion [23, 18,5] allows to derive a number of interesting results:

(i) existence of a limit distribution ( $\tau$ fixed, $L \rightarrow \infty$ ) with an algebraic tail 17]:

$$
P(\tau)=\frac{\lambda}{2 k \tau^{2}} \mathrm{e}^{-\frac{\lambda}{2 k \tau}} .
$$

(ii) Linear divergence of the first moment and exponential divergence of the higher moments [18]:

$$
\begin{aligned}
\langle\tau(k)\rangle= & \frac{L}{k} \\
\left\langle\tau(k)^{n}\right\rangle= & \left\{\sum_{m=2}^{n}(-1)^{n-m} C_{n}^{m} \frac{(m-2) !(2 m-1)}{(n+m-1) !} \mathrm{e}^{2 m(m-1) L / \lambda}\right. \\
& \left.\quad+\frac{(-1)^{n+1}}{n !}\left(2 n \frac{L}{\lambda}+n-1\right)\right\}\left(\frac{\lambda}{2 k}\right)^{n}
\end{aligned}
$$
(9) expression (7). In the inset: tail of the integrated distribution,

In [6] we have shown that these results hold for two different models of random potential in the localised regime $(L \gg \lambda)$.

In order to test the analytical results in the above mentioned regime it is convenient to choose a model suitable for numerical simulations. For this purpose we have considered the case where the random potential is given by a sum of delta functions of the same weight $v$, randomly dropped on the half line with an average density $\rho$ (the so-called Frish and Lloyd model) . The equations that

\footnotetext{
* This model coincides with the Gaussian model (Halperin model) considered in [6] in the limit of a high density of impurities $(v \ll k \ll \rho)$.
}

FIG. 1. Time delay distribution in the localised regime $L \gg \lambda$. Comparison between the numerical calculation and numerical and analytical.

describe the evolution of the phase take a discrete form which can be implemented conveniently in a numerical simulation. The distribution of the time delay that we have obtained numerically in this way is in perfect agreement with equation (7) as soon as the high energy regime is reached. For example we compare on figure 11 the analytical expression (7) with the corresponding numerical result for a regime $\rho \ll v \ll k$. The simulation was carried out for a system with $10^{5}$ impurities of weight $v=1$, distributed with an averaged density $\rho=0.1$. The energy considered corresponds to $k=10$ which is related to a localisation length $\lambda=\frac{8 k^{2}}{\rho v^{2}}=8000$. The ratio $L / \lambda=125$ is sufficiently large for the limit distribution to be reached. The numerical calculation is based on a statistics of 50000 values. It shows that the algebraic tail is well reproduced by (7) for 2000 times the typical value $\tau_{\text {typ }}=200$. Let us stress that there is no adjustable parameter to fit the numerics. The only parameter entering in the analytical expressions is the localisation length which is known for each kind of disorder.

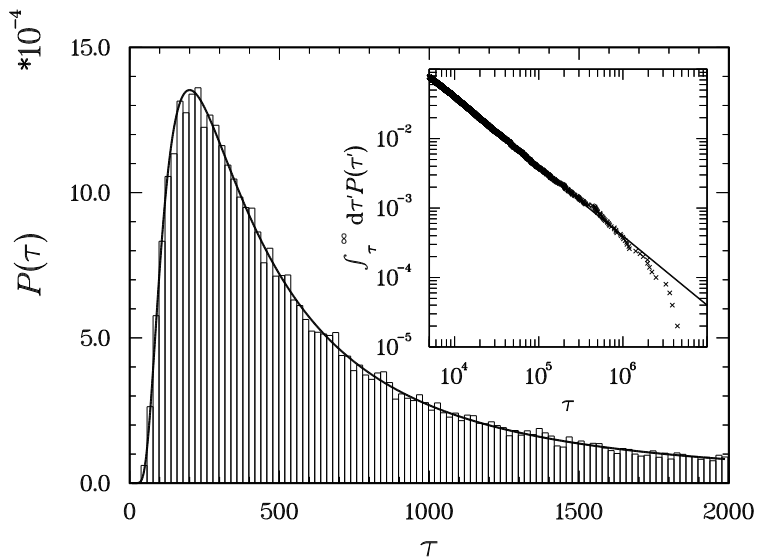

The derivation of the statistical properties of $\tau$ given above allows one to understand the universality of the result but on the other hand does not shed much light on the physical mechanisms which are responsible for the occurence of an algebraic tail. In the following we propose a physical picture based on the existence of resonances that explains the leading exponential behaviour of the moments. The starting point is to realize that the reflection of the incident wave on the random potential can in fact be viewed as a resonance tunneling process [9, 2]. Indeed there exists a representation of the time delay as a superposition of resonances of energy $E_{\alpha}$ and width $\Gamma_{\alpha}$ in the form 12] 


$$
\tau(E)=2 \sum_{\alpha} \frac{\Gamma_{\alpha} / 2}{\left(E-E_{\alpha}\right)^{2}+\Gamma_{\alpha}^{2} / 4} .
$$

Obviously the dominant contribution $\tau \simeq \frac{4}{\Gamma_{\alpha}}$ is achieved when $E$ is in a window of width $\Gamma_{\alpha}$ centered at $E_{\alpha}$, and this will occur with probability $\frac{\Gamma}{\Delta}$ where $\Delta$ is the mean level spacing. In order to estimate the width we may assume that a discrete level $E_{\alpha}$ localised at $x_{0}$ will be broadened by its coupling to the continuum of states through the end point $x=L$. We may therefore set $\Gamma \sim \mathrm{e}^{-2 \gamma_{L}\left(L-x_{0}\right)}$ where $\gamma_{L}$ is the Lyapunov exponent in the finite system. Assuming that $x_{0}$ is uniformily distributed on $[0, L]$ and decorrelated from $\gamma_{L}$ one obtains the estimate:

$$
\left\langle\tau^{n}\right\rangle \sim \int_{0}^{L} \frac{\mathrm{d} x_{0}}{L} \int \mathrm{d} \gamma_{L} p\left(\gamma_{L}\right) \frac{\Gamma}{\Delta} \frac{1}{\Gamma^{n}} .
$$

Since $\xi(L)=L \gamma_{L}$ defined previously is a Gaussian process, the distribution of the finite size Lyapunov exponent $\gamma_{L}$ is [1]

$$
p\left(\gamma_{L}\right)=\sqrt{\frac{L}{2 \pi \gamma}} \mathrm{e}^{-\frac{L}{2 \gamma}\left(\gamma_{L}-\gamma\right)^{2}} .
$$

One finally obtains

$$
\left\langle\tau^{n}\right\rangle \sim \mathrm{e}^{2 n(n-1) L / \lambda} .
$$

A more refined derivation [22] allows to recover the gross behaviour of the pre-exponential factor. This demonstrates that this particular behaviour of the moments has his origin both in the exponentially small widths of the resonances and in the fluctuations of the Lyapunov exponent for the finite size sample.

This physical picture shows that the problem of the time delay distribution related to the question of quantum relaxation in disordered systems. In this respect, the fact that $P(\tau)$ is a broad distribution is rather puzzling. Indeed it was recently argued that at large time, the probability distribution of various quantities, such as the conductance of one-dimensional disordered sample, exhibits a log-normal tail [19]. In order to clarify this point, instead of considering as before the regime $\tau$ fixed $L \rightarrow \infty$ which leads to (7), we have studied for fixed $L$ the tail of the distribution in the limit $\tau \rightarrow \infty$. In order to extract the asymptotic behaviour, it is convenient to consider the characteristic function $\phi(p, L)=\int_{0}^{\infty} \mathrm{d} \tau \mathrm{e}^{-2 k p \tau} P(\tau ; L)$ given in [18]. If the conjugated variable $p$ is chosen in a range $\gamma \mathrm{e}^{-\gamma L} \ll p \ll \gamma \mathrm{e}^{-\sqrt{\gamma L}}$, the characteristic function exhibits the following behaviour

$\phi(p, L) \simeq 1-\frac{2 \sqrt{\pi} \mathrm{e}^{-\frac{\gamma L}{2}}}{(2 \gamma L)^{3 / 2}} \ln \gamma / p\left[1+O\left(\frac{\ln \gamma / p}{\gamma L}\right)\right] \mathrm{e}^{-\frac{\ln ^{2} \gamma / p}{8 \gamma L}}$,

which suggests the existence of a log-normal tail for the distribution

$$
P(\tau ; L) \sim \exp -\frac{1}{8 \gamma L} \ln ^{2}(2 k \gamma \tau)
$$

in the range $\mathrm{e}^{\gamma L} \gg 2 k \gamma \tau \gg \mathrm{e}^{\sqrt{\gamma L}}$. Although we were not able to derive the behaviour of the distribution when $\mathrm{e}^{\gamma L} \ll 2 k \gamma \tau$, the fact that the most divergent part of the moments grow like $\mathrm{e}^{2 n^{2} L / \lambda}$ suggests that the distribution is still log-normal.

As an aside remark let us mention that a log-normal distribution of the time delay also occurs in the study of the random mass Dirac model at the critical point $E=0$ 21]. There, the authors provide a representation of $\tau$ which is similar to equation (6), except that the drift term is absent. This problem can also be analysed by using the approach given in [18,5].

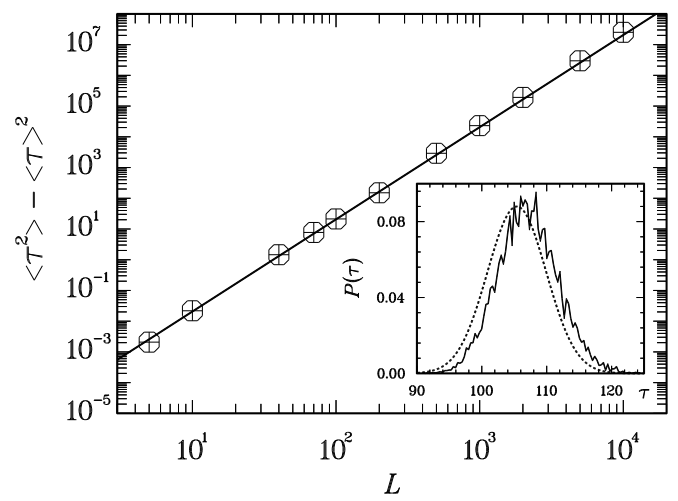

FIG. 2. Second cumulant of the time delay in the ballistic regime $L \ll \lambda$. Comparison between numerical results and the analytical result $\left\langle\tau^{2}\right\rangle-\langle\tau\rangle^{2}=\frac{\rho v^{2}}{6\left(k^{2}-\rho v\right)^{2}} L^{3}$. the parameters are $v=0.001, \rho=100$ and $k=1$. In the inset: time delay distribution for $L=100$ ( $10^{4}$ impurities).

At this stage we have only considered a localised regime for which the size of the system is large compared to the localisation length $L \gg \lambda$. Another interesting case is the ballistic one characterised by $L \ll \lambda$. In this situation, the dimensionless variable $\gamma L$ which arises in (6) is small compared to 1 and the argument of the exponential typically remains small compared to 1 , which allows one to expand the exponential. The resulting expression for the time delay is given by a linear functional of a Gaussian quantity and has itself Gaussian fluctuations characterised by a first moment $\langle\tau\rangle=\frac{L}{k}$ and a second cumulant $\left\langle\tau^{2}\right\rangle-\langle\tau\rangle^{2} \simeq \frac{4 \gamma}{3 k^{2}} L^{3}$. We have checked numerically these results with the delta impurity model. We have considered a regime where it reproduces the high energy features of the Gaussian model: $v \ll k \ll \rho$ and $\left(k^{2}-\rho v\right) \gg\left(\rho v^{2}\right)^{2 / 3}$. In this regime one has to take into account the averaged value of the disorder $\rho v$ and replace $k$ by $\sqrt{k^{2}-\rho v}$ in all previous expressions: the localisation length is thus given by $\lambda=\frac{8\left(k^{2}-\rho v\right)}{\rho v^{2}}$ 
and the moments of $\tau$ now read: $\langle\tau\rangle=\frac{L}{\sqrt{k^{2}-\rho v}}$ and $\left\langle\tau^{2}\right\rangle-\langle\tau\rangle^{2} \simeq \frac{\rho v^{2}}{6\left(k^{2}-\rho v\right)^{2}} L^{3}$. In figure 2 we compare the numerical result to the Gaussian distribution where the parameters are given by the previous expressions. The calculation is performed for a ratio $L / \lambda \simeq 1.410^{-3} .10000$ values of $\tau$ were calculated.

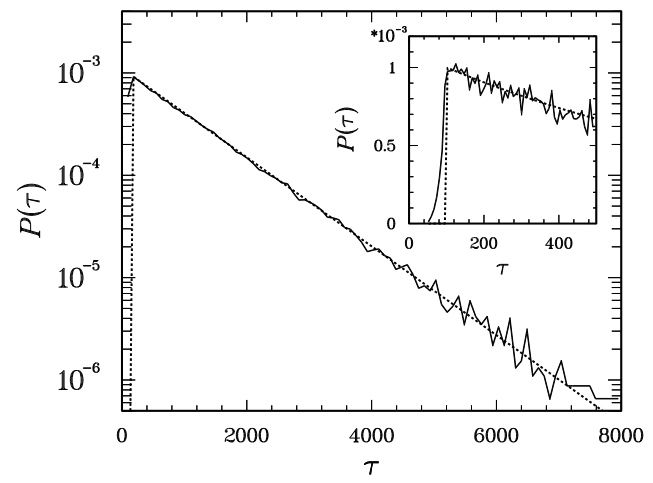

FIG. 3. Time delay distribution in the localised regime $L \gg \lambda$ at low energy.

Let us close the paper with some remarks on the lowenergy regime. In this case one is more sensitive to the precise nature of the disorder, therefore one can guess that universality must break down. The distribution of the time delay will now depend on the nature of disorder. As an illustration let us consider the delta impurity model; we predict [22], in the low density regime $k \ll \rho \ll v$, an exponential tail for the distribution:

$$
P(\tau) \simeq k \rho \mathrm{Y}\left(\tau-\frac{1}{k v}\right) \mathrm{e}^{-k \rho\left(\tau-\frac{1}{k v}\right)}
$$

where $\mathrm{Y}(x)$ denotes the Heaviside function. We have checked that this expression is in very good agreement with the numerical results. The numerical computation was performed for $v=1, \rho=0.1, k=0.01$ for 1000 impurities. The resulting distribution presented on figure 3 is based on 50000 data sets. The behaviour at the origin is more subtle than the one given above, nevertheless (16) gives the correct scale on which the distribution vanishes at the origin. Another indication that universality breaks down is the fact that within this same model, the low energy regime with a high density of impurities $k \ll v \ll \rho$ leads to different distributions, though still characterised by an exponential tail.

\section{Acknowlegments}

We thank David Dean, Yan Fyodorov, Spyros Evangelou, Alexander O. Gogolin, Leonid Pastur and Marc Yor for interesting discussions. We thank Marie-Thérèse Commault for the realization of figures. $\ddagger$ e-mail: comtet@ipno.in2p3.fr

$\dagger$ e-mail: texier@ipno.in2p3.fr

[1] T. N. Antsygina, L. A. Pastur and V. A. Slyusarev, Sov. J. Low Temp. Phys. 7, 1 (1981).

[2] M. Ya. Azbel, Solid State Commun. 45, 527 (1983).

[3] P. W. Brouwer, K. M. Frahm and C. W. Beenakker, Phys. Rev. Lett. 78, 4737 (1997).

[4] M. Büttiker, Phys. Rev. B 27(10), 6178 (1983).

[5] A. Comtet, C. Monthus and M. Yor, J. Appl. Prob. 35, 255 (1998).

[6] A. Comtet and C. Texier, J. Phys. A: Math. Gen. 30, 8017 (1997).

[7] L. Eisenbud, PhD thesis, Princeton (1948); E. P. Wigner, Phys. Rev. 98(1), 145 (1955).

[8] W. G. Faris and W. J. Tsay, SIAM J. Appl. Math. 54, 443 (1994).

[9] U. Frisch, C. Froeschle, J.-P. Scheidecker and P.-L. Sulem, Phys. Rev. A 8, 1416 (1973).

[10] Y. V. Fyodorov and A. D. Mirlin, Int. J. of Mod. Phys. B 8(27), 3795 (1994).

[11] Y. V. Fyodorov and H.-J. Sommers, Phys. Rev. Lett. 76, 4709 (1996).

[12] Y. V. Fyodorov and H.-J. Sommers, J. Math. Phys. 38, 1918 (1996).

[13] V.A. Gopar, P.A. Mello and M. Büttiker, Phys. Rev. Lett. 77, 3005 (1996).

[14] J. Heinrichs, J. Phys. Cond. Mat. 2, 1559 (1990).

[15] A. M. Jayannavar, G. V. Vijayagovindan, and N. Kumar, Z. Phys. B - Cond. Mat. 75, 77 (1989).

[16] S. K. Joshi and A. M. Jayannavar, preprint condmat/9712249 (1997).

[17] Professor L. Pastur pointed out that, in an infinite system, the norm of the wave function is distributed with a similar law. Cf. $\S 13.3$ of the book: I. M. Lifshits, S. A. Gredeskul and L. A. Pastur (1988), Introduction to the theory of disordered systems, John Wiley \& Sons (1988). This distribution also occurs in the context of classical diffusion in a random potential : J.-P. Bouchaud, A. Comtet, A. Georges and P. le Doussal, Ann. of Phys. (NY) 201, 285 (1990).

[18] C. Monthus and A. Comtet, J. Phys. I (France) 4, 635 (1994); G. Oshanin, A. Mogutov and M. Moreau, J. Stat. Phys. 73, 379 (1993).

[19] B. A. Muzykantskii and D. E. Khmelnitskii, Phys. Rep. 288, 259 (1997).

[20] F. T. Smith, Phys. Rev. 118, 349 (1960).

[21] M. Steiner, Y. Chen, M. Fabrizio and A. O. Gogolin, preprint cond-mat/9806220 (1998).

[22] C. Texier and A. Comtet, in preparation; C. Texier, PhD thesis, Université Paris 6 (1999).

[23] M. Yor, Adv. Appl. Prob. 24, 509 (1992); M. Yor (Ed.), Exponential functionals and principal values related to Brownian motion, Madrid: Biblioteca de la revista matemática iberoamericana (1997). 